\title{
Karsthydrologische Forschungen in den Höhlen Ungarns
}

\author{
Von Hubert Kessler, Budapest
}

Mit 3 Figuren und 6 Abbildungen im Text

Obwohl Ungarn nicht zu den bekannten großen Karstländern gehört, haben die hiesigen speleologischen und besonders karsthydrologischen Forschungen bedeutende Erfolge zu verzeichnen.

Dies ist zwei Umständen zu verdanken: Einerseits befindet sich im nordungarischen Karstgebiet bei Aggtelek eine der größten Tropfsteinhöhlen der Welt, deren wissenschaftliche Erforschung natürlich für alle Speleologen sehr anziehend ist, andererseits konzentrieren sich die großen Rohstofflager auf die ausgedehnten, wasserarmen Karstgebiete. Die zu diesen Rohstoffen gebundene und in großem Aufstieg befindliche Industrialisierung hat natürlich große Wasseransprüche, die eben an Mangel von Oberflächenwasser nur aus der Tiefe zu decken sind.

Diesem Umstand ist es zu verdanken, daß sich ein Teil der Hydrologen und Geologen unter bedeutender amtlicher Unterstützung den speleologischen und karsthydrologischen Forschungen widmen konnte. Einige dieser Forschungen, die Anspruch auf allgemeines Interesse oder internationale Zusammenarbeit haben, sollen im folgenden kurz erörtert werden.

\section{Die aktiven wasserführenden Höhlen Ungarns}

Die größten aktiven Höhlen liegen im nordungarischen Karstgebiet, nahe der tschechoslowakischen Grenze. Diese sind hauptsächlich aus mitteltriadischem Kalk aufgebaut und grenzen südlich an tertiäres Gebiet an, dessen Oberflächenwasser bei der Berührungszone durch offene Schlinger in das Kalkmassiv eindringt. Die Erosionswirkung des Wassers wird durch das mitgeschwemmte Material, hauptsächlich Quarzkieselschotter, bedeutend gesteigert, was die Ausbildung mächtiger Hohlräume besonders begünstigt.

Die größte der nordungarischen Höhlen ist die weltbekannte Aggteleker-Tropfsteinhöhle mit über $20 \mathrm{~km}$ langen erschlossenen und vermessenen Gängen. Der Verbindungsgang mit der auf tschechoslowakischem Gebiet liegenden Domicahöhle wurde durch den Verfasser nach Überwindung von zwei Syphonen entdeckt. 
Diese Höhle hat drei gut gesonderte Etagen. Die oberste ist nicht mehr wasserführend und hat den größten Tropfsteinreichtum. Die mittlere, etwa $11 \mathrm{~m}$ tiefer liegende Etage ist zeitweise wasserführend. Das Wasser ergießt sich stellenweise durch unterirdische Schlinger in die unterste, zum größten Teil noch unerschlossene juvenile Höhle und tritt in einer mächtigen Karstquelle bei Jósvafő zutage.

Seit dem Jahre 1954 wurden noch weitere 1-5 km lange Höhlen durch die Forscher Balázs, Holly, Jakucs, Maucha und den Verfasser entdeckt. Es gelang zum Teil durch künstliche Erweiterung der Wasserschlinger, zum Teil durch bei den Quellen gesprengte Stollen in diese durch vorherige systematische hydrologische Beobachtungen vermuteten Höhlen zu dringen.

Zeitweilig wasserführende Höhlen gibt es noch im Kalkplateau des Bükk-Gebirges und im südlichen Teil Ungarns, bei Abaliget.

Besondere Beachtung verdienen die thermalwasserführenden Höhlen, deren größte in Tapolca (Balaton-See) im tertiären Kalk liegt. Das aus größerer Tiefe, aus untergelagertem Dolomit stammende stagnierende Karstwasser von $18^{\circ} \mathrm{C}$ bildet unterirdische Seen von etwa $250 \mathrm{~m}$ Länge. In den letzten Jahren wurden durch Taucher noch über $300 \mathrm{~m}$ unter Wasser liegende Hohlräume erschlossen und vermessen. Diese Hohlräume erstrecken sich noch weiter, doch setzte die Kapazität der Preßluftflaschen ihrer Erschließung vorläufig Grenzen.

Eine andere thermalwasserführende Höhle liegt in der Nähe von Miskolc. Hier entspringen Karstquellen von $30^{\circ} \mathrm{C}$, die jetzt für ein unterirdisches Bad benutzt werden.

Bei Budapest wurden mehrere Höhlen entdeckt, die noch im Tertiär thermalwasserführend waren, jedoch seit der Hebung der Kalkschollen über den jetzigen Karstwasserspiegel kamen und so trockengelegt wurden. In diesen Höhlen sind einzigartige Ablagerungen des früheren Thermalwassers in Form von Aragonit, Alabaster, Barit usw. zu sehen.

Erwähnenswert sind die Untersuchungen, die die besondere Reinheit, Staubfreiheit der Höhlenluft in den wasserführenden Höhlen feststellten. Zum Vergleich führen wir auch den Kerngehalt (unter $10 \mu$ ) der Luft außerhalb der Höhlen an:

Tabelle

\begin{tabular}{l|c|c}
\hline \multirow{2}{*}{ Untersuchungsort } & \multicolumn{2}{|c}{ Kerngehalt in 1 Liter Luft } \\
& im Freien & in der Höhle \\
\hline Seehöhle bei Miskolc & 1500 & 311 \\
Seehöhle bei Tapolca & 8700 & 210 \\
Bachhöhle bei Abaliget & 1100 & 95
\end{tabular}


Auf Grund dieser auffallenden Ergebnisse wurden in diesen Höhlen versuchsweise an Asthma bronchitis erkrankte Personen unter ärztlicher Aufsicht untergebracht, um die klinische Bedeutung der Höhlenluft genauer kennenzulernen. Die Untersuchungen sind noch nicht abgeschlossen, die bisherigen Ergebnisse sollen jedoch vielversprechend sein.

\section{Die Frage der Versickerung in Karstgebieten}

Die Frage der Versickerung, besonders des Versickerungsprozentes, in Karstgebieten ist dort von besonderer Bedeutung, wo das Karstwasser zu wasserwirtschaftlichen Zwecken benutzt wird, wo also die ausbeutbare Menge mit praktisch genügender Genauigkeit bestimmt werden muß.

Auf Grund einer 25jährigen Beobachtungsreihe von Quellenschüttungen konnte die Gesetzmäßigkeit zwischen Niederschlag und Schüttung erforscht und dabei die grundliegende Wirkung der Vegetationsperioden erkannt werden. In Fig. 1 ist der durchschnittliche Versickerungsprozent in den 12 Monaten des Jahres graphisch dargestellt.

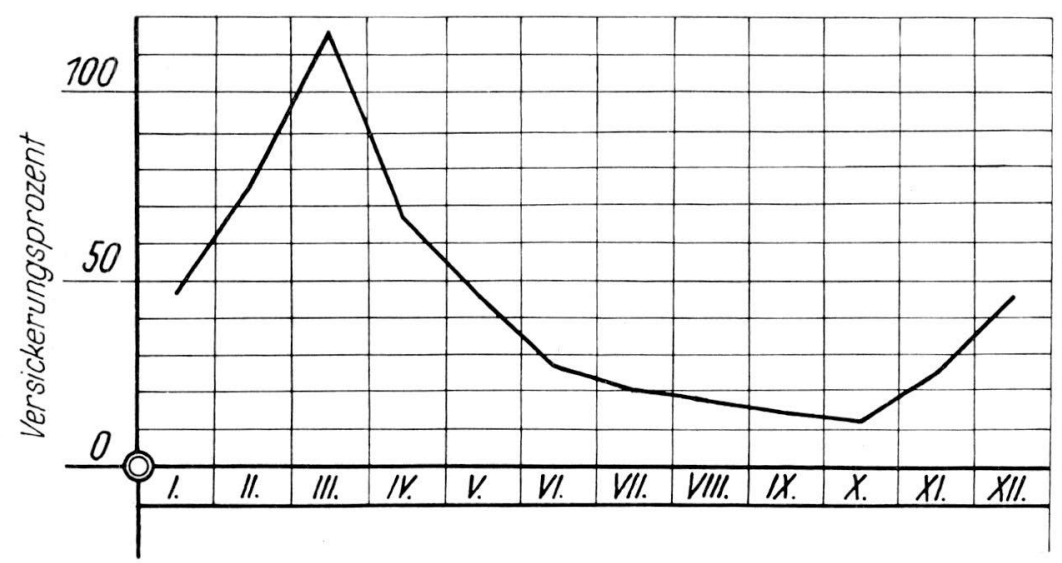

Fig. 1. Das durchschnittliche monatliche Versickerungsprozent in den ungarischen Karstgebieten.

Der über hundertprozentige Wert im März ist auf den in Form von Schnee akkumulierten Niederschlag vorhergehender Monate zurückzuführen.

In Zusammenhang mit diesen Forschungen wurde in Höhlen die Tropfintensität bei Stalaktiten untersucht. Tropfintensität nennen wir die in einer Stunde getröpfelte Wassermenge in Kubikzentimetern ausgedrückt. 
Es wurden in verschiedenen Höhlen täglich gemessene Mensuren oder automatisch registrierende Instrumente unter einigen Stalaktiten aufgestellt und die Tropfintensität seit 5 Jahren gemessen. Es war von Bedeutung, festzustellen, wie der Niederschlag in den verschiedenen Monaten die Tropfintensität beeinflußt. Die in Fig. 2 dargestellte Kurve zeigt die durch $1 \mathrm{~mm}$ Niederschlag verursachte Steigerung der Tropfintensität. Die Kurve hat eine auffallende Ähnlichkeit mit der in Fig. 1 dargestellten. Der Einfluß der Vegetationsperioden ist auch in diesem Fall eindeutig. Während zum Beispiel im März die Tropfintensität durch $1 \mathrm{~mm}$ Niederschlag um 9,5 $\mathrm{cm}^{3} /$ Stunde steigt, hat der im August oder September gefallene Niederschlag praktisch keine Wirkung.

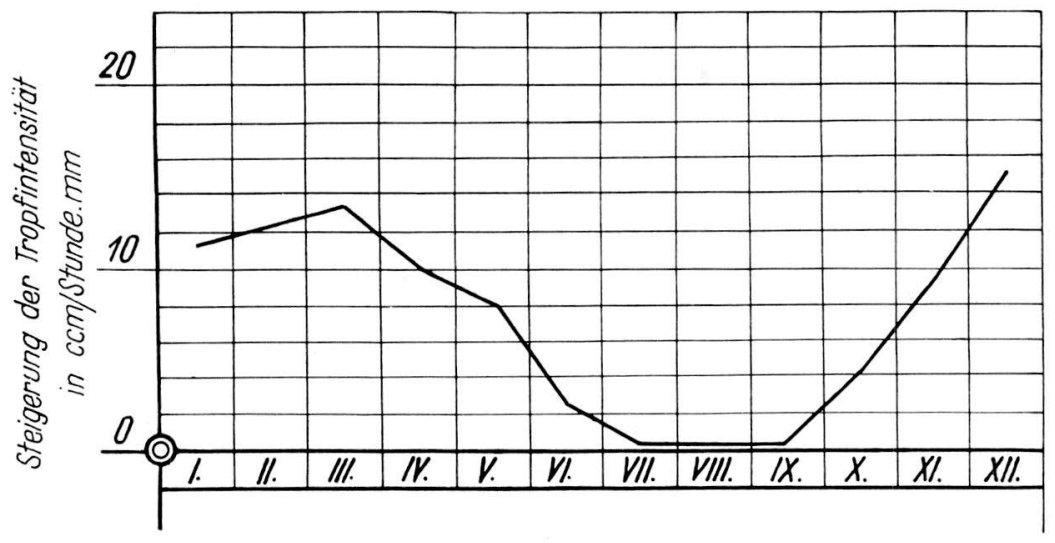

Fig. 2. Die Steigerung der Tropfintensität in Höhlen durch $1 \mathrm{~mm}$ Niederschlag.

Zur Feststellung der Versickerungsgeschwindigkeiten wurden in mehreren Karstgebieten künstliche Berieselungen vorgenommen, und in den darunter befindlichen Höhlen wurde der Anstieg der Tropfintensität gemessen. Es wurden durchschnittlich im Dachsteinkalk 6,7-9,1 m/Stunde und im Eozänkalk 2,4-4,7 m/Stunde Versickerungsgeschwindigkeiten gemessen.

Um das Einzugsgebiet einzelner Karstquellen zu erörtern, wurden zwischen Wasserschlinger und Quellen Färbungs- und Salzungsversuche durchgeführt, und dabei wurde auch die Fließgeschwindigkeit der unterirdischen Wasserläufe festgestellt. Auffallend war, daß sich die Fließgeschwindigkeit bei neben größerer Schüttung wiederholten Versuchen nicht im gleichen Verhältnis vergrößerte. Hieraus ist der Schluß zu ziehen, daß in solchen Fällen der unterirdische Quellgang 
nicht ein unter Druck stehender, voll ausgefüllter Kanal ist, sondern ein weiter Hohlraum, der bei größerer Schüttung eine Vergrößerung des sogenannten ,,benetzten Querschnittes" und deshalb eine vielmals vergrößerte Wasserführung bei verhältnismäßig geringerer Steigerung der Fließgeschwindigkeit erlaubt. Es ist also eine Möglichkeit gegeben, durch Geschwindigkeitsmessungen bei verschiedenen Schüttungen auf die durchschnittlichen Querschnittsverhältnisse der unbekannten, wasserführenden Höhlengänge zu folgern.

Gute Erfolge mit zahlenmäßigen Ergebnissen hatten wir bei Salzungen und elektrischen Widerstandsmessungen durch Feldgeräte zu verzeichnen.

In Zusammenhang mit den Tropfintensitätsversuchen kamen wir zu der Erkenntnis, daß die im Querschnitt von Tropfsteinen sichtbaren Ringe möglicherweise ,Jahresringe“ sind. Der im Winterhalbjahr versickernde kältere Niederschlag ist aufnahmefähiger für Kohlensäure und löst deshalb mehr $\mathrm{Ca} \mathrm{CO}_{3}$ als der warme sommerliche Niederschlag, der dagegen mehr Farbstoffe und aus dem Humus stammende organische Substanzen lösen kann. Das kältere Tropfwasser verursacht daher reinere, weiße Ringe, während das sommerliche Getröpfel dunklere, braune Ringe bildet. Ein Ringpaar spiegelt deshalb den Versickerungsvorgang eines Jahres.

Es werden jetzt weitere Forschungen vorgenommen, um den Rhythmus der Ringe, ähnlich wie bei der Dendrochronologie, festzustellen, um eine eventuelle Periodizität zu erkennen. Mit deren Hilfe ist es möglich, die Niederschlagsverhältnisse der vergangenen Jahrtausende aufzuklären, was natürlich auch für die langfristige hydrologische Vorhersage von Bedeutung sein kann.

\section{Die Änderung der Ionenkonzentration des Karstwassers}

Sowohl für den Speleologen als auch für den Karsthydrologen ist es von Bedeutung aus der chemischen Beschaffenheit der Karstwässer sowie ihrer Änderung auf die noch unbekannten Karstgänge schließen zu können. Den Speleologen interessiert es, ob hinter einer Karstquelle großräumige, weite Höhlengänge voraussetzbar sind, der $\mathrm{Hy}$ drologe, der für die Wasserwirtschaft arbeitet, möchte wissen, ob bei der Quelle große Schwankungen der Schüttung oder eine ausgeglichene Wasserlieferung zu erwarten sind. Meistens fehlt es an langjährigen Meßreihen, die solche Fragen beantworten können. Es gibt aber Möglichkeiten, auf Grund einiger Messungen der Schüttung und der Ionenkonzentration wichtige Folgerungen schließen zu können. 
Die Stabilität der chemischen Zusammensetzung des Karstwassers ist durch die elektrische Widerstandsmeßmethode leicht und schnell zu kontrollieren. Zu diesem Zweck wurden leichte Feldgeräte konstruiert, mit denen die Änderung des elektrischen Widerstandes eines Karstgerinnes auch unter den schwierigsten Verhältnissen an Ort und Stelle gemessen werden kann.

Es war der Zweck einer Untersuchung, festzustellen, wie sich der elektrische Widerstand des Wassers von der Schwinde bis zur Quelle ändert und welche Faktoren die Änderung beeinflussen.

Eine besonders geeignete Gelegenheit zu diesen Untersuchungen bietet das ausgiebig erschlossene Höhlensystem bei Aggtelek, wo der Lauf des Wassers von der Schwinde bis in die Nähe der Quelle gut verfolgbar ist. Hier wurde bei verschiedenen Niederschlagsverhältnissen der elektrische Widerstand des Wassers bei den Schwinden, in der Höhle und bei der Quelle gemessen. Die schematische Skizze (Fig. 3) soll einen Überblick über das Höhlensystem und die Meßstellen geben.

Die Höhle bzw. das karsthydrographische System bildet sich aus dem etwa $12 \mathrm{~km}$ langen Hauptgang, der in der Tschechoslowakei mit der Schwinde Ördöglyuk beginnt. Eine zweite, größere Schwinde ist noch Domica. Auf ungarischem Gebiet ist die erste Schwinde Csernaitó, dann Acheron, von wo das Wasser in $300 \mathrm{~m}$ langem Lauf in breitem Gang in den im Hauptgang fließenden Bach Styx fließt. Eine größere Schwinde ist Bábalyuk, doch ist der Verbindungsgang mit dem Höhlensystem noch unbekannt, deshalb auf der Skizze nicht ganz ausgezogen. Die Schwinde Török Mecset führt durch einen sehr engen, $1200 \mathrm{~m}$ langen Arm in die Haupthöhle. Durch einen breiten, $3000 \mathrm{~m}$ langen Arm gelangt das bei der Schwinde Retekág eintretende Wasser bis zum Styx, der sich schließlich etwa $1000 \mathrm{~m}$ vor der Quelle durch eine unterirdische Schwinde in das noch unerschlossene, äußerst eng und deshalb unbefahrbare untere Höhlensystem ergießt und dann bei der Jósva-Quelle zutage tritt.

Das bei den Schwinden einfließende Wasser ist im allgemeinen sehr weich: Härtegrad 2,2-4,4, was einem elektrischen Widerstand von $16500 \mathrm{Ohm}$. cm-9450 Ohm. cm entspricht. Je höher die Ionenkonzentration des Wassers ist, um so kleiner ist der elektrische Widerstand.

Bemerkenswert ist, daß der Widerstand des bei der RetekágSchwinde gemessenen Wassers bei etwa $2000 \mathrm{~m}$ unterirdischem Lauf von $8450 \mathrm{Ohm}$. cm auf $6718 \mathrm{Ohm}$. cm sinkt, während das bei der TörökMecset-Schwinde einfließende Wasser von 9357 Ohm. cm auf einer kürzeren Strecke $(1200 \mathrm{~m})$ auf $3843 \mathrm{Ohm}$. cm $\left(8,4\right.$ d. H. $\left.{ }^{\circ}\right)$ sinkt. In anderen Worten ist in diesem Fall die spezifische ,Erhärtung“ des 


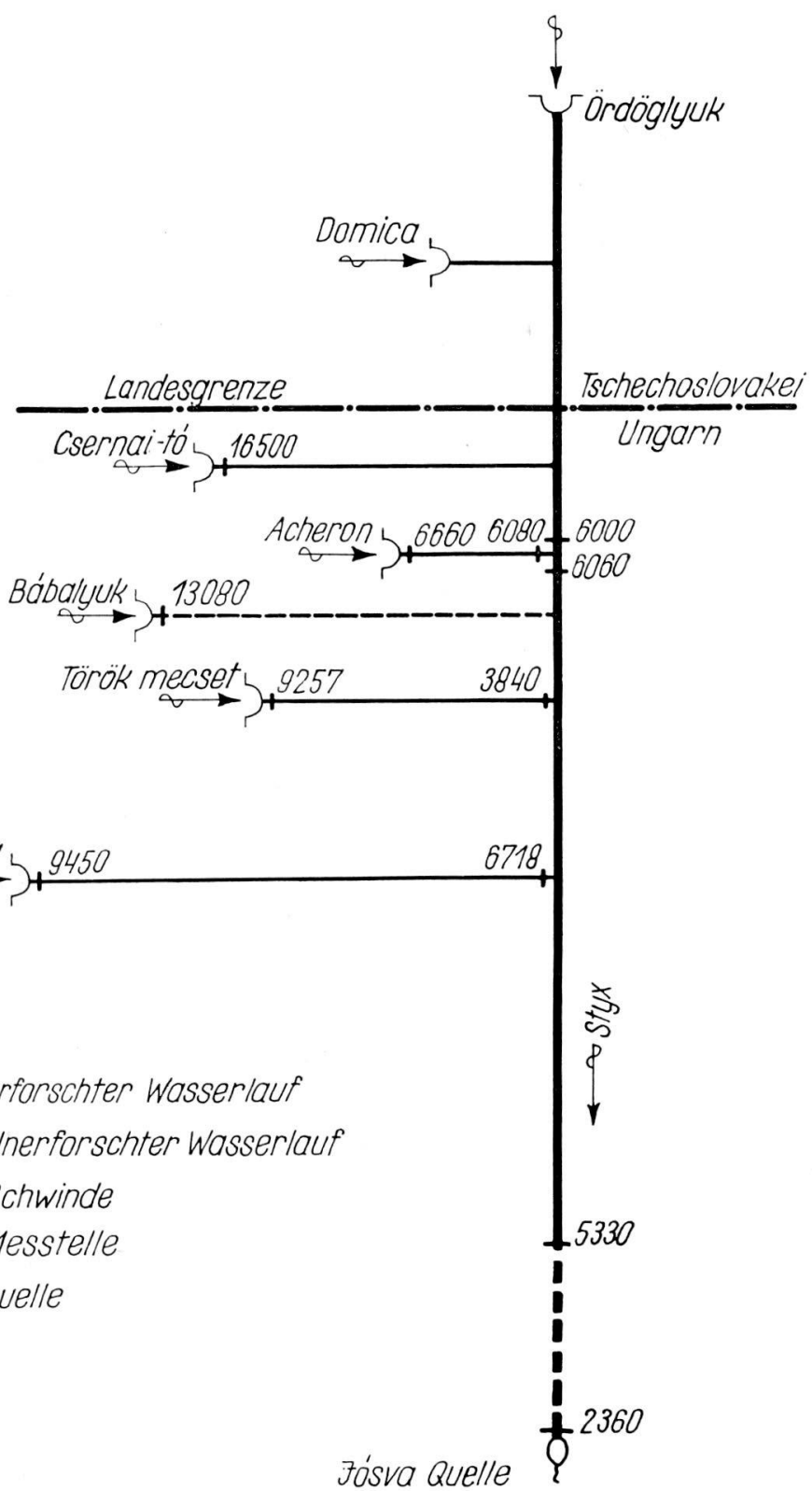

Fig. 3. Schematische Darstellung der unterirdischen Wasserläufe im Aggteleker Höhlensystem. Die Zahlen bedeuten den elektrischen Widerstand des Wassers in Ohm. cm. 
Wassers bedeutend größer als im vorigen. Es ist dies mit dem kleineren Querschnitt des letzteren Seitenarmes verbunden. Damit ist auch zu begründen, daß der elektrische Widerstand des Styx im Hauptgang kaum eine nennenswerte Änderung zeigt. Nur im letzten Teil seines Flusses, zwischen der unterirdischen Schwinde und der Quelle, sinkt der Widerstand des Wassers von 5392 Ohm. cm auf 2358 Ohm. cm. Es ist dies damit zu begründen, daß der Bach sich hier in einem sehr engen Spaltensystem bewegt, wo er sich mit einer verhältnismäßig großen, Lösungsmöglichkeiten bietenden Felsenfläche berührt.

Es ist also durch Messung des elektrischen Widerstandes eines Karstgerinnes die Möglichkeit gegeben, auf die Querschnittsverhältnisse in den noch unbekannten Höhlenpartien zu schließen.

Die auf der schematischen Skizze angeführten Zahlen bedeuten die elektrischen Widerstandswerte in Ohm. cm.

\section{Erschließungsmöglichkeiten unbekannter Höhlen}

Wie schon erwähnt, wurden in Ungarn mehrere größere wasserführende Höhlen auf Grund längerer systematischer Beobachtungen von Karstquellen künstlich erschlossen. Es soll nun kurz angedeutet werden, welche hydrologischen Untersuchungen dazu beitragen, um die Existenz eines noch unerschlossenen Höhlensystems nachweisen zu können. Voraussetzung ist natürlich, daß die geologischen und morphologischen Verhältnisse schon genügend geklärt sind.

Unseren Erfahrungen gemäß ist es ein für die Bildung größerer Höhlensysteme besonders günstiger Umstand, wenn das Einzugsgebiet einer Karstquelle zum Teil aus nichtverkarstetem Boden besteht, der aus härteren Gesteinarten als der Kalkstein aufgebaut ist. In diesem Falle vergrößert das eingeschwemmte Geschiebe bedeutend die Erosionswirkung des Wassers im Karst.

Allgemein bekannt sind die Verfahren, mit denen die Zugehörigkeit einer Schwinde zu einer Karstquelle nachgewiesen wird. Der Zusammenhang kann durch Färbung, durch Salzung und durch die von österreichischen Forschern ausgearbeitete Sporentriftung nachgewiesen werden. Wenn es sich aber nicht nur um den Nachweis der Verbindung handelt, sondern wir auch nähere Daten über die vermutlichen Größenverhältnisse des Höhlengerinnes erhalten wollen, dann bewährt sich am besten die Salzungsmethode, verbunden mit elektrischer Widerstandsmessung. In diesem Falle bekommen wir nämlich ein zahlenmäßig auswertbares, genaues Bild vom ganzen Ablauf der „,Salzwolke“, von der Ankunft der ersten Spuren, der höchsten Kon- 
zentration und vom Ablauf der Triftung. Eine verhältnismäßig kurze Zeitspanne zwischen den ersten und letzten Salzspuren deutet auf einen unter Druck liegenden, ganz mit Wasser ausgefüllten Gang, während ein längeres Zeitintervall mit mehreren elektrischen Widerstandsminima auf einen breiteren, durch unterirdische Seen unterbrochenen Höhlenbach weist.

Besonders wichtig ist es, die Triftungen bei verschiedenen Schüttungen zu wiederholen und dann die gewonnenen Daten, besonders die Fließgeschwindigkeiten, zu vergleichen. Wenn bei mehrfacher Schüttung die Fließgeschwindigkeit nicht ebenso steigt, dann ist auch mit der Wahrscheinlichkeit eines breiteren, durch Wasser nicht ganz ausgefüllten Höhlenganges zu rechnen.

Diese Triftungsuntersuchungen sind aber allein noch nicht geeignet, die Höhlensysteme mit genügender Wahrscheinlichkeit nachzuweisen. Öfters ist es auch nicht möglich eine Wasserschwinde zu finden. Es müssen deshalb komplexe Untersuchungsmethoden angewendet werden. Zu diesen gehört die systematische Messung der Schüttung, der Temperatur, des Chemismus und des elektrischen Widerstandes des Wassers.

Bei der Auswertung der Messungsreihen sind folgende Erfahrungen bzw. Überlegungen im Auge zu behalten.

Ein Höhlensystem mit großen, weiträumigen Gängen führt das nach einem größeren Niederschlag oder nach Schneeschmelze eingedrungene Wasser ohne Stauung ungehindert zur Quelle und verursacht dort bald nach dem Niederschlag ein Hochwasser. Da eben wegen der ungehinderten Abflußmöglichkeit keine Stauung, Akkumulierung des Wassers im Karst möglich ist, fällt die Schüttungskurve nach der Niederschlagsperiode wieder steil ab. Der Lauf des Höhlenbaches verlangsamt sich und setzt einen großen Teil des mitgeführten Schwemmaterials im Bachbett ab. Diese Lehmablagerungen werden bei der nächsten Hochwasserperiode durch die große Fließgeschwindigkeit wieder aufgewirbelt und trüben das Quellwasser. Die bei Hochwasser eintretenden Trübungen sind also ebenfalls günstige Voraussetzungen für den Nachweis größerer Höhlenräume.

In dem Fall, daß das unterirdische Wasser in engen, wenn auch hohen Klüften, Spalten fließt, tritt bei durch eine größere Wassermenge bedingtem größerem ,,benetztem" Querschnitt ein verhältnismäßig größerer Reibungswiderstand auf, der die Fließgeschwindigkeit unter bestimmten Grenzen hält. Es ist deshalb eine größere Rückstauung im Karst möglich, das Hochwasser meldet sich bei der Quelle nicht so schnell und stark, und der Ablauf ist auch verzögert. Bei ge- 
ringerer Wasserführung verlangsamt sich die Geschwindigkeit nicht sehr, es kommt nicht zur Lehmablagerung, und deshalb ist in solchen Fällen eine Trübung der Quelle bei Hochwasser nicht oder nur selten zu beobachten.

Ähnlich sind die Temperaturschwankungen und die Schwankungen des elektrischen Widerstandes auszuwerten. Enge, durch Wasser ganz ausgefüllte Hohlräume gleichen die Temperatur mehr aus als weite Räume, in denen das Wasser in einem Bachbett fließt, und ebenso ist eine größere Lösungsmöglichkeit geboten, die natürlich den elektrischen Widerstand beeinflußt.

Zusammengefaßt: Große, mit Trübungen verbundene Schwankungen der Schüttung, der Temperatur und des elektrischen Widerstandes deuten auf verhältnismäßig weite Hohlräume.

Einige Worte noch über den Chemismus des Wassers in Verbindung mit den Höhlen. Das durchschnittliche Ca/Mg-Verhältnis des Wassers interessiert uns hauptsächlich. Wie bekannt, begünstigen Dolomiteinlagerungen oder dolomitische Kalke die Höhlenbildung nicht. Das Verhältnis der dolomitischen Komponente im Karst zeigt sich im $\mathrm{Ca} / \mathrm{Mg}$ Verhältnis des Wassers. Großer Magnesiumgehalt deutet auf überwiegend dolomitische Lagerungen. Es ist also ein großes $\mathrm{Ca} / \mathrm{Mg}-\mathrm{Ver}-$ hältnis günstiger als ein kleineres.

Wenn die Möglichkeit besteht, den elektrischen Widerstand des Wassers bei einer oder mehreren Schwinden zu messen, gibt der Vergleich mit dem dazugehörigen Quellenwasser auch gute Stützpunkte zur Beurteilung der unbekannten Höhlengänge.

Ein noch genaueres Bild über die vermuteten aktiven Höhlen können wir erhalten, wenn wir unsere Meßergebnisse mit solchen vergleichen können, die uns bei schon bekannten unterirdischen Karstgerinnen zur Verfügung stehen.

Bei solchen Vergleichsmöglichkeiten ist auch der Inhalt an gelöstem Sauerstoff in Betracht zu ziehen. Höhlen, in denen der Bach mit verhältnismäßig großer freier Oberfläche und durch viele Wasserfälle intensiv mit der Höhlenluft in Berührung kommt, ermöglichen einen größeren Sauerstoffgehalt des Wassers.

$\mathrm{Zu}$ einigermaßen sicheren Prognosen müssen Meßreihen zur Verfügung stehen, die wenigstens ein Jahr durchgeführt wurden und die verschiedene Niederschlagsverhältnisse erfassen. Auf Grund solcher Vorarbeiten konnten in Ungarn schon mehrere Kilometer lange wasserführende Höhlen durch bei der Quelle oder der Schwinde gesprengte Stollen künstlich erschlossen werden, die unsere Voraussetzungen bestätigten. 


\section{Die praktische Bedeutung für die Wasserversorgung}

Die vorhin erwähnten Untersuchungen sind auch für die Wasserwirtschaft von Bedeutung, mit dem Unterschied, daß die für den Höhlenforscher günstigen Umstände von dem Wasserwirtschaftler als ungünstig gewertet werden. Für die Wasserwirtschaft sind Karstquellen von Bedeutung, die eine ausgeglichene Schüttung, also kleine Schwankungen, haben. Bei diesen ist keine Trübung zu befürchten, und die bakteriologischen Verhältnisse sind infolge der besseren Filterwirkung günstiger. Besonders ein kleiner, auf Dolomitlagerungen hinweisender Ca/Mg-Quotient ist bei solchen Quellen sehr vorteilhaft, die zur Wasserversorgung einbezogen werden sollen. Die karsthydrologischen Untersuchungen sind also die gleichen wie die beim Speleologen, nur bei der Auswertung müssen gegenteilige Standpunkte berücksichtigt werden.

Die aus speleologischen Gründen durchgeführten Untersuchungen sind also jedenfalls von großem Wert für die Wasserwirtschaftler, aber oft kommt es vor, daß die wasserwirtschaftlichen Erschließungsarbeiten speleologische Erfolge bringen. Zur Sicherung der Wasserversorgung auf Karstgebieten wurden in Ungarn schon mehrere Schächte ausgehoben, mit denen das in großer Tiefe, in der Nähe von Bruchzonen in Spalten und Höhlen fließende Karstwasser erschlossen wurde. Zur Nachweisung dieser unter Wasserdruck stehenden Höhlen sind natürlich große speleologische Erfahrungen nötig, die sich die Forscher in den zugänglichen Höhlen erwerben.

Es mußten große technische Schwierigkeiten überwunden werden, da diese Schächte tief unter den Karstwasserspiegel reichen müssen und auch die Pumpstationen wasserdicht ausgebildet unter diesem Niveau ausgebaut werden, um das Wasser mit möglichst großer Depression zu gewinnen.

Die speleologische Erforschung eines Karstgebietes ist auch dann von Bedeutung, wenn die Karstquellen zu Trinkwasserversorgungszwecken benutzt werden und deshalb die unterirdischen Verbindungen im hygienischen Schutzgebiet erforscht werden müssen, wie dies zum Beispiel im Bükk-Gebirge in Verbindung mit der Wasserversorgung der Stadt Miskole unter großem Aufwand durchgeführt wurde.

Die Zusammenarbeit zwischen Speleologen und Wasserwirtschaftlern ist - wie dies durch zahlreiche praktische Erfahrungen bewiesen wurde-jedenfalls sehr nützlich und ist in Ungarn gesichert. Die speleologischen Aufgaben werden durch die Gesellsehaft für Karstund Höhlenforschung, die karsthydrologischen Forschungen durch das Forschungsinstitut für Wasserwirtschaft in Budapest durchgeführt. 


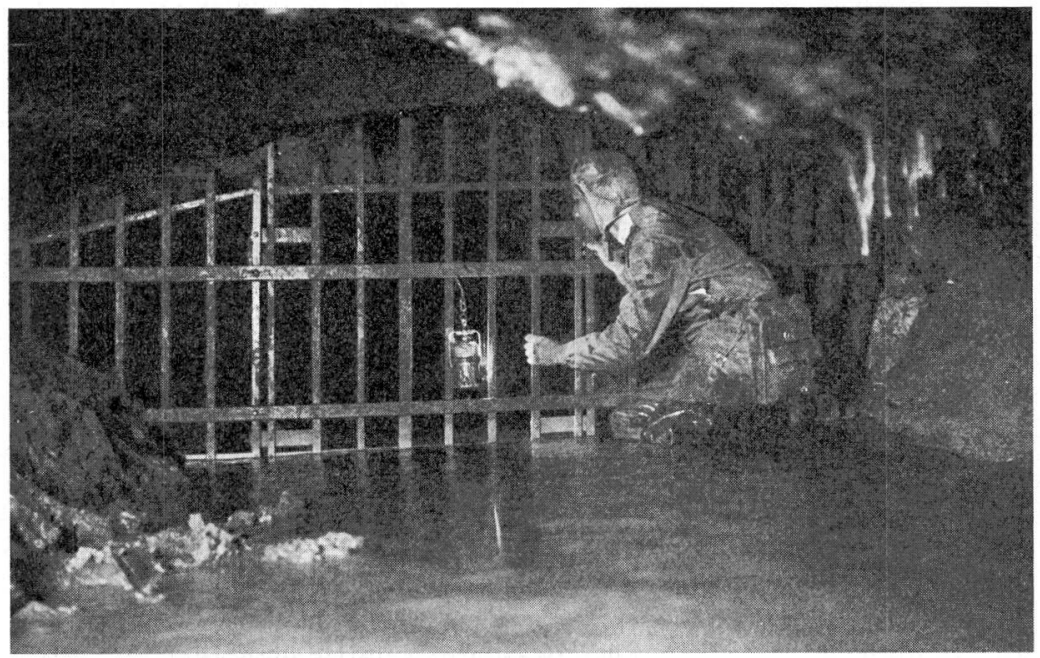

Abb. 1. Die unterirdische Landesgrenze zwischen Ungarn und der Tschechoslowakei im Aggteleker Höhlensystem. Phot. Kessler.

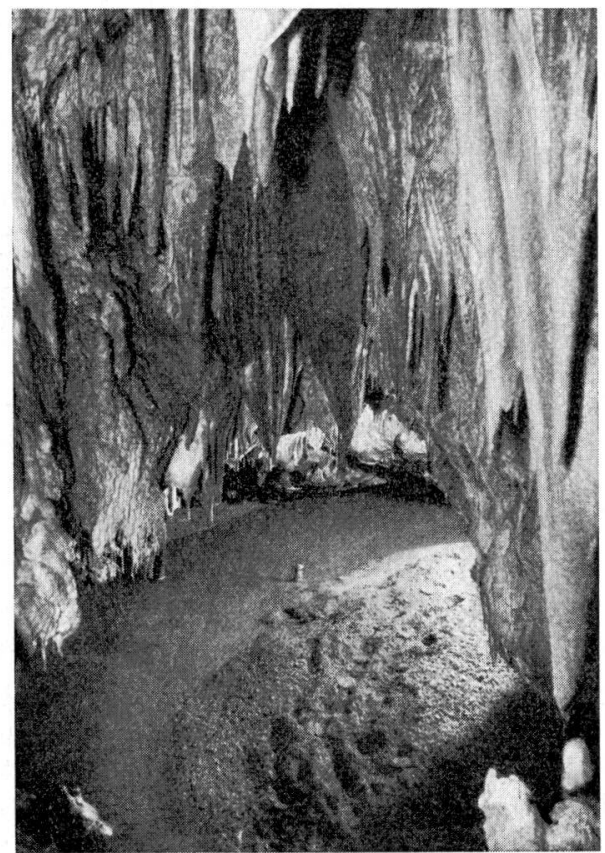

Abb. 2. Unterirdischer Bachlauf in der Béke-Höhle (Aggtelek). Phot. Kessler. 


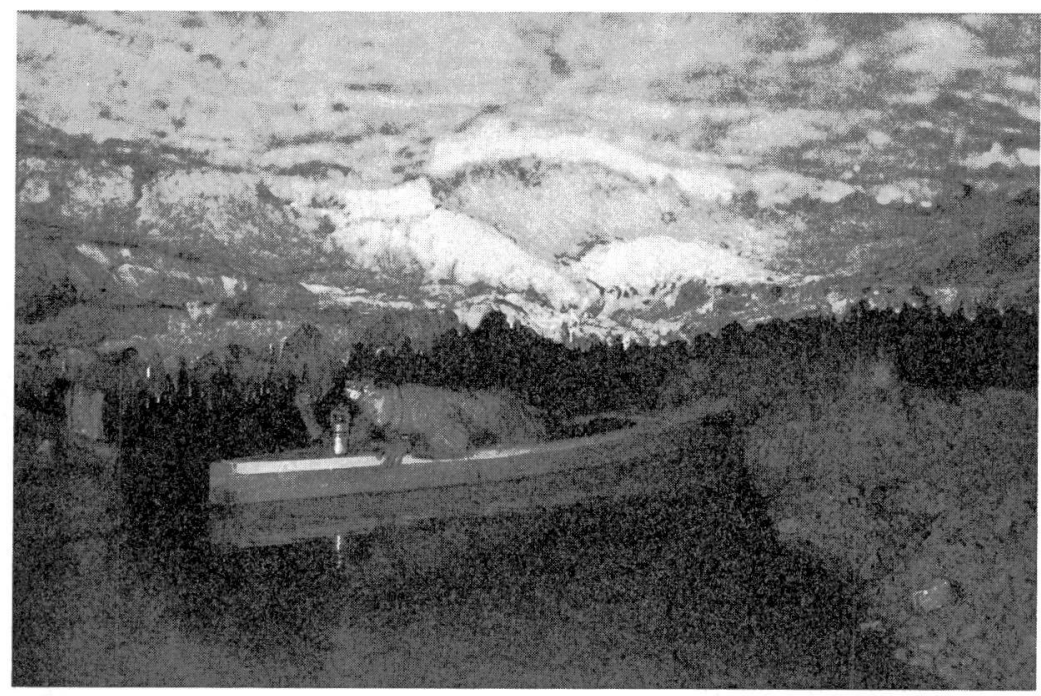

Abb. 3. Erforschung des „Styx“ in der Nähe der Syphone. Phot. Kessler.

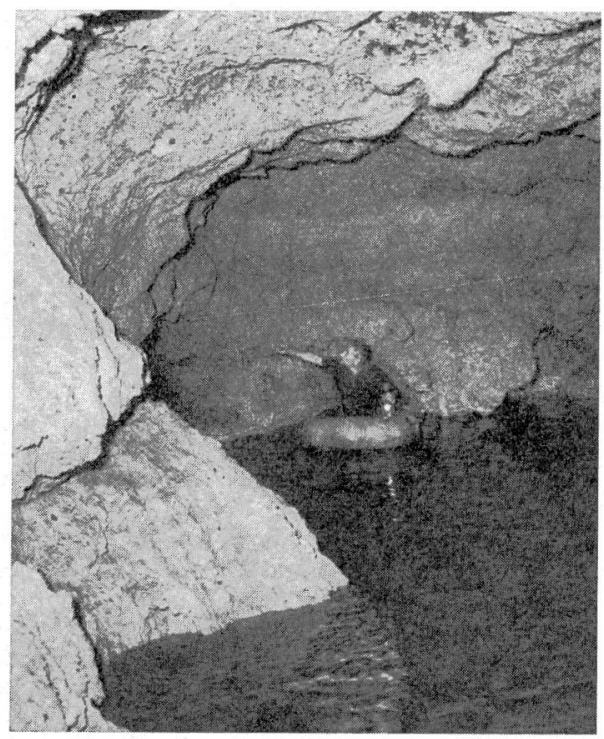

Abb. 4. Durch Abpumpen freigewordener Hohlraum unter einer Karstquelle bei Tata. Phot. M. T. I. 


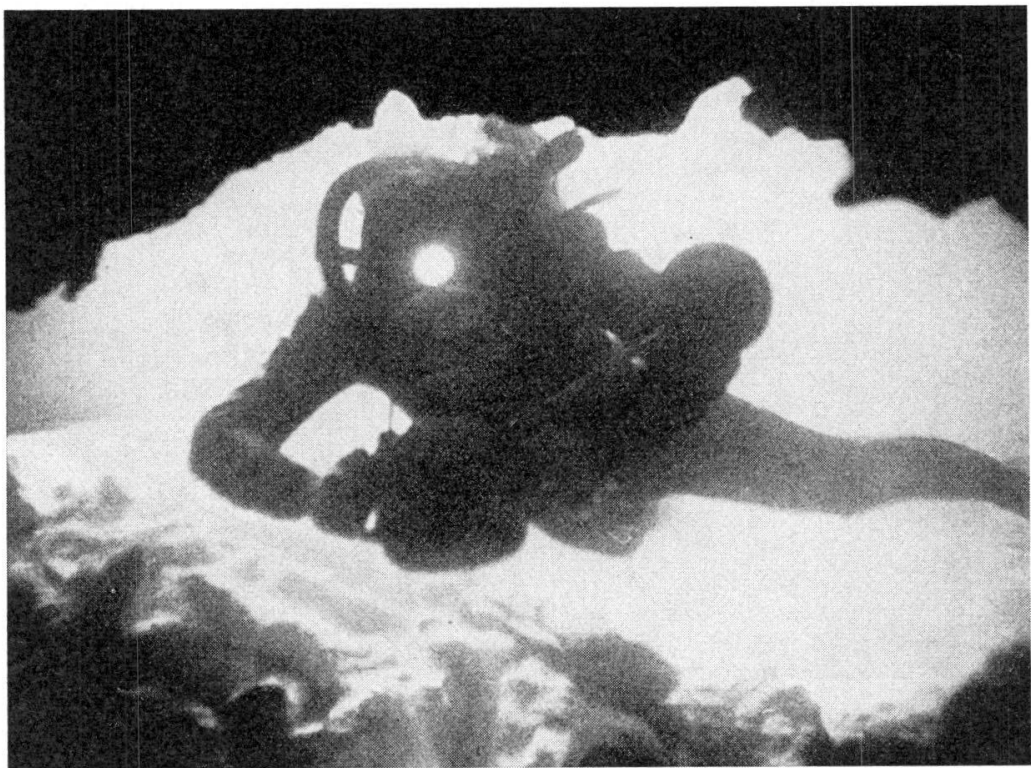

Abb. 5. Unterwasserforschung in der Seehöhle bei Tapolca. Phot. Hajdu.

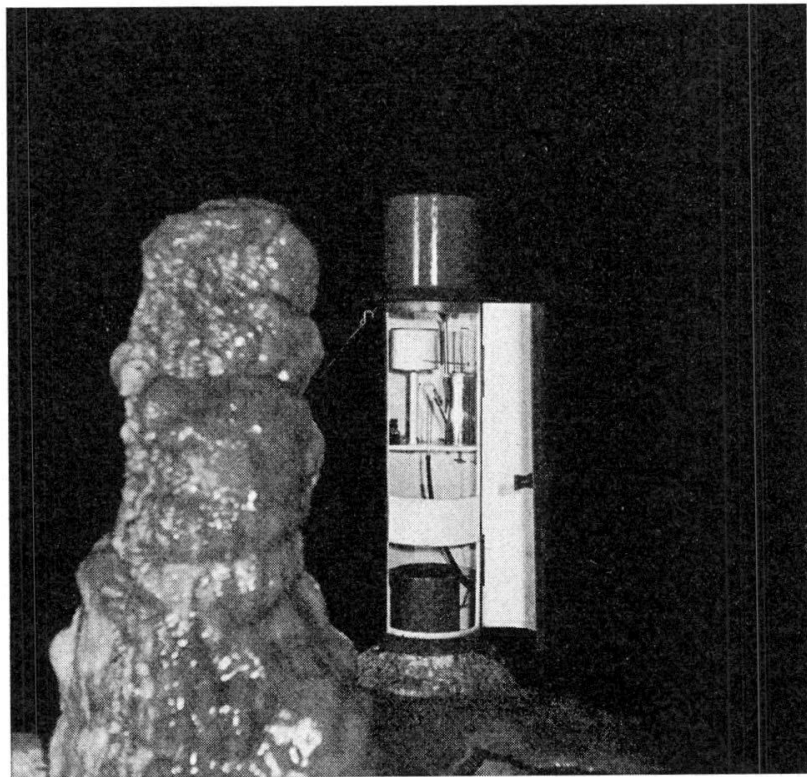

Abb. 6. Registrierung der Tropfintensität in Höhlen. Phot. Rádai. 


\section{ZUSAMMENFASSUNG}

Trotzdem Ungarn nicht zu den großen Karstländern gehört, werden hier großzügige speleologische und karsthydrologische Forschungen durchgeführt. Erstens befindet sich in Ungarn eine der größten Tropfsteinhöhlen der Welt, zweitens ist ein großer Teil der Rohstoffe und der damit verbundenen Industrie an Karstgebiete gebunden, die besondere Wasserversorgungsprobleme stellen.

Die größten wasserführenden Höhlen sind in Nordungarn. Die bekannteste ist die $22 \mathrm{~km}$ lange Aggteleker-Höhle, aber außerdem gibt es dort mehrere 1-5 km lange, neu erschlossene Höhlen, die durch künstliche Eingriffe auf Grund jahrelanger hydrologischer Beobachtungen entdeckt wurden. Besonders zu erwähnen sind die aktiven Thermalhöhlen mit $30^{\circ}$ Wasser. In einer Höhle wurde durch Taucher ein $300 \mathrm{~m}$ langer Syphon entdeckt und vermessen. In anderen werden versuchsweise speleotherapeutische Behandlungen durchgeführt.

Durch Auswertung von jahrzehntelangen Messungsreihen wurde eine praktisch brauchbare Formel zur Errechnung des Versickerungsprozentes in Karstgebieten niedergelegt. In mehreren Höhlen wird der Einfluß des Niederschlages auf die Tropfintensität gemessen. Die Deutung der sogenannten , ,Jahresringe“ der Tropfsteine ergibt Daten über die Niederschlagsverhältnisse vergangener Jahrtausende, die auch für die Periodenforschung wertvoll sind. Es wurde in mehreren Höhlen die Änderung der Ionenkonzentration der Wasserläufe gemessen und die Korrelation mit dem Querschnitt der Höhlen festgestellt. Auf Grund komplexer Messungen bei Karstquellen ist die Möglichkeit gegeben, auf noch unbekannte Höhlensysteme $\mathrm{zu}$ schließen. Es wurden so in letzter Zeit mehrere Höhlen künstlich erschlossen.

\section{SUMMARY}

Although Hungary does not belong to the large Karst countries, extensive speleologic and karst-hydrologic investigations are carried out. On the one hand, Hungary is one of the largest stalactyte caves in the world, on the other hand the majority of raw materials and the connected industries are linked with Karst regions which pose particular water supply problems.

The largest water supplying caves are in the North of Hungary. The best known cave is the Aggtelek cave with a length of $22 \mathrm{~km}$, but there are numerous other, recently disclosed caves of a length of $1-5 \mathrm{~km}$, which were discovered by way of artificial means and on the basis of many years of hydrologic observations. Of particular interest are the active thermal caves with waters of $30^{\circ} \mathrm{C}$. In one of these latter a diver discovered and measured a siphon of a length of $300 \mathrm{~m}$. By way of experiment, speleotherapic treatments were applied in some of these caves.

By calculation of decades of series of measurings an applicable formula was established for the calculation of the percent of seepage in the Karst regions. In several of these caves the influence of precipitation on the intensity of stalactyte formation was measured. The indication of the socalled ,,year-rings" in the stalactytes furnishes data concerning precipitation of bygone millenaries, which are also valuable for the investigation of periods. In several caves the changes in ion concentration of the water currents was measured and the correlation with the cross section of the 
caves was determined. On the basis of complex measurements in Karst sources the possibility of disclosing hitherto unknown cave systems arises. In this manner, recently several caves were artificially discovered.

\section{LITERATUR}

JAKUCS, L. - Nuovi metodi di studio e risultati delle ricerche nelle grotte d'Ungheria. Rassegna Speleologica Italiana. Como. Anno XII. Fasc. 1: 3-10.

Kessler, H. - (1956) Das aggteleker Höhlengebiet (Nordungarn). Fremdenverkehrsamt der Stadt Miskolc. 1-72.

- (1957) - Estimation of subsurface water resources in karstic regions. Assemblée Générale de Toronto (I.A.S.H.) Tome II : 199-206.

Maucha, L. (1959) - Das Nachweisen von Höhlensystemen. Karszt- és Barlangkutatás. Budapest. 89-96. 